Environmental and atmospheric data was obtained from the EPA, including PM2.5 and ozone concentration, temperature, residual wind, relative humidity, and barometric pressure. The average values of each factor 10 days prior to patient visit was calculated. Univariate and multivariate models were built in order to study the association of these variables with lupus disease activity. The models were adjusted for age, sex, income, racial distribution, and rural vs. urban patient residence. Multivariate logistic regression was used to identify significant determinants associated with lupus flares. Regression was performed for each organ flare outcome. Regression inference was based on generalized estimating equations (GEE) to account for the time repeated outcomes.

Results Rash, serositis, hematologic, and joint flares were statistically significantly associated $(\mathrm{p}<0.00 .5)$ with an increase in temperature in univariate and multivariate analysis. Renal flares were negatively associated with increases in temperature $(\mathrm{p}<0.05)$ in univariate and multivariate analysis.

PM2.5 concentration was significantly associated $(p<0.001)$ with rash, joints, serositis, neurologic, pulmonary, and hematologic flares in univariate and multivariate analysis.

Ozone concentration, residual wind, and relative humidity were significantly associated with lupus flares in univariate analysis only, while barometric pressure had no associations.

Conclusions There is a strong association between changes in PM2.5 concentration and temperature 10 days prior to patient visit and organ specific lupus activity at the visit. These data could add an important aspect to lupus trials, the outcomes of which may be affected by so far unrecognized environmental factors, and ultimately it could allow predictive modelling of lupus flares which would revolutionize the approach to treatment.

Funding Source(s): The Hopkins Lupus Cohort was funded by AR 69572

\section{CARDIOVASCULAR RISK FACTORS IN A SYSTEMIC LUPUS ERYTHEMATOSUS COHORT FORM COLOMBIA}

${ }^{1}$ Sebastian Herrera*, ${ }^{2}$ Juan C Díaz-Coronado, ${ }^{3}$ Deicy Hernandez Parra, ${ }^{4}$ Laura BetancurVásquez, ${ }^{4}$ Jorge Lacouture-fierro, ${ }^{4}$ Daniel Gonzalez-Hurtado, ${ }^{4}$ Laura Uribe-Arango, ${ }^{4}$ Maria Fernanda Saavedra-Chacón, ${ }^{4}$ Santiago Monsalve-Yepes, ${ }^{4}$ Sebastián Guerra-Zarama, ${ }^{4}$ José David Serna-Giraldo, ${ }^{4} J u a n$ David López-López, ${ }^{4} J u l i a ́ n$ Barbosa-Arana, ${ }^{2}$ Ricardo A PinedaTamayo. 'Grupo información clínica, ARTMEDICA; ${ }^{2}$ Grupo de Información Clínica Artmedica IPS; ${ }^{3}$ ARTMEDICA; ${ }^{4}$ Departamento medicina interna, Universidad CES

\subsection{6/lupus-2019-|sm.298}

Background Systemic lupus erythematosus (SLE) is a chronic and multisystemic autoimmune disease. Higher prevalence of traditional and disease associated risk factors, such as corticosteroids and accelerated atherosclerosis due to chronic inflammation, result in an increased cardiovascular risk. Age and

Abstract 298 Table 1 Bivariate model with cardiovascular risk as dependent variable

\begin{tabular}{|c|c|c|c|}
\hline Variable & Crude OR & IC $95 \%$ & $P$ value \\
\hline \multicolumn{4}{|l|}{ Age in years } \\
\hline $36-45$ & 1,7 & $1,13-2,64$ & 0,001 \\
\hline $46-55$ & 3,5 & $2,41-5,06$ & 0,001 \\
\hline $56-65$ & 8,2 & $5,44-12,55$ & 0,001 \\
\hline$>66$ & 12,3 & $7,00-21,82$ & 0,001 \\
\hline \multicolumn{4}{|l|}{ Civil status } \\
\hline Married & 0,9 & $0,74-1,22$ & 0,733 \\
\hline Single & 1,2 & $1,05-1,59$ & 0,01 \\
\hline \multicolumn{4}{|l|}{ Education years } \\
\hline $0-5$ & 1 & & \\
\hline $6-11$ & 2,9 & $2,07-4,22$ & 0,001 \\
\hline$>12$ & 1,6 & $1,23-2,25$ & 0,001 \\
\hline Positive ds-DNA & 0,7 & $0,57-0,96$ & 0,025 \\
\hline Hypocomplementemia & 0,6 & $0,51-0,85$ & 0,001 \\
\hline \multicolumn{4}{|c|}{ Antimalarial use } \\
\hline Chloroquine & 0,81 & $0,62-1,05$ & 0,11 \\
\hline Hydroxychloroquine & 0,85 & $0,62-1,16$ & 0,31 \\
\hline \multicolumn{4}{|l|}{ Years of antimalarial use } \\
\hline $1-5$ & 0,91 & $0,64-1,29$ & 0,61 \\
\hline $6-10$ & 0,47 & $0,34-0,64$ & 0,001 \\
\hline$>11$ & 0,44 & $0,31-0,63$ & 0,001 \\
\hline \multicolumn{4}{|c|}{ Other medication } \\
\hline Corticosteroids & 2,2 & $1,32-3,80$ & 0,02 \\
\hline Cyclophosphamide & 0,5 & $0,24-1,33$ & 0,19 \\
\hline Mycophenolate & 1,1 & $0,64-2,06$ & 0,62 \\
\hline Rituximab & 1,3 & $0,49-3,47$ & 0,58 \\
\hline
\end{tabular}


corticosteroid use have been described as cardiovascular risk factors but there is controversy surrounding antimalarials as a protective factor. Our objective is to analyze associated factors with the presentation of cardiovascular events such as high blood pressure (HBP), acute myocardial infarction (AMI), stroke and thromboembolic disease (TED)

Methods A cross-sectional study was done with 1175 records of patients with SLE that fulfilled either ACR 1997 of SLICC 2012 classification criteria that had been in medical care between 2015 and 2017 in a rheumatology specialized institution in six cities of Colombia. We describe sociodemographic, clinical and immunoserological characteristics and a comparative analysis was done with chi2 and Mann Whitney's U with a combined outcome of cardiovascular disease obtaining an OR of crude associations that were adjusted for several variables

Results Women represented $91 \%$ of the cohort with a median age of 44 years (IQR 21) and 8 years of disease duration (IQR 11) with a mean age at diagnosis of 32 years, 5,4\% were active smokers and $15 \%$ had smoked in the past. Cardiovascular events were found in $32 \%$ of the patients with HT as the most common. Other cardiovascular outcomes such as stroke, TED and AMI were infrequent with a prevalence of $3.3 \%, 2.9 \%$ and $2 \%$ respectively. In bivariate analysis, age $>36$ years and corticosteroid use were associated with a significantly higher risk, while the use of antimalarials for more than 6 years was found to protect for cardiovascular risk with no difference between chloroquine and hydroxychloroquine use (table 1)

Conclusions Our cohort is comparable with other SLE cohorts regarding the frequency of cardiovascular events. Up to $32 \%$ of the described population presented a cardiovascular event and arterial hypertension was the most frequent. Continuous use of antimalarials for more than 6 years has a protective effect against cardiovascular events such as arterial hypertension, stroke, acute myocardial infarction and thromboembolic disease. The benefit seen only after 6 years of continuous use probably reflects the need of a long period of time before some of the potential benefits of these medications are seen Funding Source(s): None

\section{INCREASED MORTALITY AMONG PATIENTS WITH SYSTEMIC LUPUS ERYTHEMATOSUS AFTER HYDROXYCHLOROQUINE DISCONTINUATION}

${ }^{1}$ Antonio Avina-Zubieta*, ${ }^{2}$ April Jorge, ${ }^{3}$ Mary A DeVera, ${ }^{4} \mathrm{Na} \mathrm{Lu},{ }^{4}$ John Esdaile, ${ }^{5}$ Hyon Choi. ${ }^{1}$ Arthritis Research Canada, Univeristy of British Columbia; ${ }^{2}$ Division of Rheumatology, Allergy, and Immunology, Massachusetts General Hospital; ${ }^{3}$ University of British Columbia; ${ }^{4}$ Arthritis Research Canada; ${ }^{5}$ Massachusetts General Hospital

\subsection{6/lupus-2019-Ism.299}

Background Hydroxychloroquine (HCQ) is near-universally recommended for patients with SLE. Use of this medication has previously been associated with a substantial survival benefit among SLE patients. We aimed to determine the potential temporal association between HCQ discontinuation and allcause and cardiovascular disease (CVD) mortality.

Methods We conducted a population-based case-control study using an administrative health database including the entire population in the province of British Columbia, Canada (>5 million individuals). We identified cases with SLE who died and each case was matched on age, sex, and SLE disease duration with living controls with SLE. We used conditional logistic regression to assess the association between current use of HCQ or recent discontinuation of HCQ and the risk of all-cause and cause-specific mortality relative to remote HCQ users. Remote users were defined by a duration greater than 365 days between the last HCQ prescription and the index date (i.e., death date). Recent users had a duration less than 365 days since the last HCQ prescription and index date. Current users had active HCQ prescriptions spanning

Abstract 299 Table 1 Risk of Death with Current Usage, Non-Usage, and Recent Discontinuation compared with Remote Usage of Hydroxychloroquine among patients with Systemic Lupus Erythematosus

\begin{tabular}{|l|c|c|c|c|}
\hline All-Cause Mortality & $\begin{array}{c}\text { Cases, } \\
\text { N }\end{array}$ & $\begin{array}{c}\text { Controls, } \\
\mathbf{N}\end{array}$ & $\begin{array}{c}\text { Crude Odds } \\
\text { Ratio (95\% CI) }\end{array}$ & $\begin{array}{c}\text { Adjusted Odds } \\
\text { Ratio (95\% CI) }\end{array}$ \\
\hline Remote HCQ Users & 72 & 106 & 1.0 (reference) & 1.0 (reference) \\
\hline Recent HCQ Discontinuers & 65 & 34 & $3.03(1.77-5.17)$ & $3.78(2.07-6.91)$ \\
\hline Current HCQ users & 32 & 156 & $0.30(0.18-0.49)$ & $0.35(0.20-0.59)$ \\
\hline HCQ Non-users & 121 & 206 & $0.83(0.55-1.24)$ & $0.93(0.59-1.44)$ \\
\hline $\begin{array}{l}\text { Cardiovascular Disease } \\
\text { Mortality }\end{array}$ & & & & \\
\hline Remote HCQ Users & 17 & 28 & $1.0($ reference) & 1.0 (reference) \\
\hline Recent HCQ Discontinuers & 17 & 11 & $2.57(0.96-6.92)$ & $4.63(1.31-16.42)$ \\
\hline Current HCQ users & 9 & 44 & $0.32(0.12-0.87)$ & $0.37(0.11-1.27)$ \\
\hline HCQ Non-users & 40 & 59 & $1.11(0.51-2.44)$ & $1.15(0.45-2.99)$ \\
\hline Other Cause Mortality & & & & \\
\hline Remote HCQ Users & 35 & 52 & $1.0($ reference) & $1.0($ reference) \\
\hline Recent HCQ Discontinuers & 33 & 18 & $3.17(1.45-6.93)$ & $3.90(1.56-9.75)$ \\
\hline Current HCQ users & 13 & 72 & $0.28(0.14-0.58)$ & $0.32(0.14-0.71)$ \\
\hline HCQ Non-users & 57 & 95 & $0.89(0.49-1.64)$ & $1.18(0.29-2.37)$ \\
\hline
\end{tabular}

